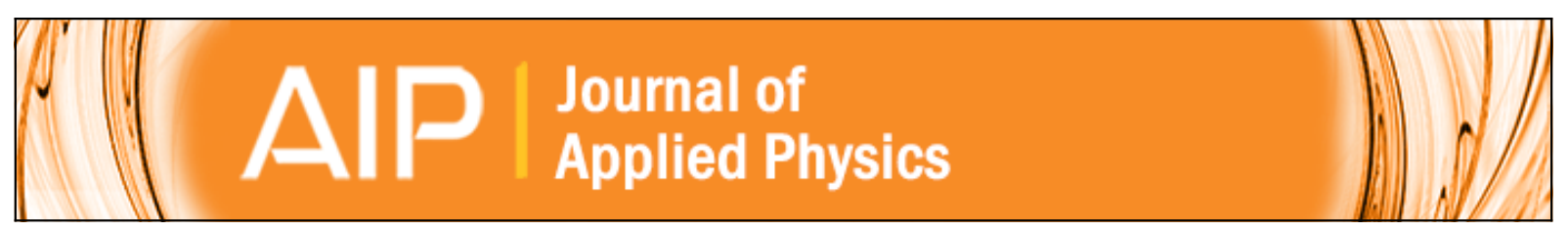

\title{
Effect of boron concentration on recombination at the p-Si-Al2O3 interface
}

Lachlan E. Black, Thomas Allen, Keith R. Mclntosh, and Andres Cuevas

Citation: Journal of Applied Physics 115, 093707 (2014); doi: 10.1063/1.4867643

View online: http://dx.doi.org/10.1063/1.4867643

View Table of Contents: http://scitation.aip.org/content/aip/journal/jap/115/9?ver=pdfcov

Published by the AIP Publishing

\section{Articles you may be interested in}

Deposition temperature independent excellent passivation of highly boron doped silicon emitters by thermal atomic layer deposited $\mathrm{Al} 2 \mathrm{O} 3$

J. Appl. Phys. 114, 094505 (2013); 10.1063/1.4819970

Interface recombination parameters of atomic-layer-deposited Al2O3 on crystalline silicon

J. Appl. Phys. 111, 073710 (2012); 10.1063/1.3700241

Controlling the fixed charge and passivation properties of $\mathrm{Si}(100) / \mathrm{Al} 2 \mathrm{O} 3$ interfaces using ultrathin $\mathrm{SiO} 2$ interlayers synthesized by atomic layer deposition

J. Appl. Phys. 110, 093715 (2011); 10.1063/1.3658246

Modulation of atomic-layer-deposited $\mathrm{Al} 2 \mathrm{O} 3$ film passivation of silicon surface by rapid thermal processing Appl. Phys. Lett. 99, 052103 (2011); 10.1063/1.3616145

Electronic and chemical properties of the c-Si/Al2O3 interface

J. Appl. Phys. 109, 113701 (2011); 10.1063/1.3587227

AIP $\left.\right|_{\text {Letters }} ^{\text {Applied Physics }}$

is pleased to announce Reuben Collins as its new Editor-in-Chief

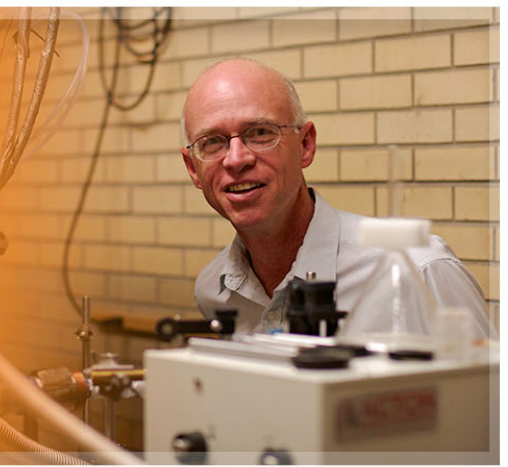




\title{
Effect of boron concentration on recombination at the $p-\mathrm{Si}_{-} \mathrm{Al}_{2} \mathrm{O}_{3}$ interface
}

\author{
Lachlan E. Black, ${ }^{1, a)}$ Thomas Allen, ${ }^{1}$ Keith R. McIntosh, ${ }^{2}$ and Andres Cuevas ${ }^{1}$ \\ ${ }^{1}$ Research School of Engineering, The Australian National University, Canberra, ACT 0200, Australia \\ ${ }^{2}$ PV Lighthouse, Coledale, NSW 2515, Australia
}

(Received 30 December 2013; accepted 23 February 2014; published online 7 March 2014)

\begin{abstract}
We examine the surface passivation properties of $\mathrm{Al}_{2} \mathrm{O}_{3}$ deposited on boron-doped planar $\langle 100\rangle$ crystalline silicon surfaces as a function of the boron concentration. Both uniformly doped and diffused surfaces are studied, with surface boron concentrations ranging from $9.2 \times 10^{15}$ to $5.2 \times 10^{19} \mathrm{~cm}^{-3}$. Atmospheric pressure chemical vapor deposition and thermal atomic layer deposition are used to deposit the $\mathrm{Al}_{2} \mathrm{O}_{3}$ films. The surface recombination rate of each sample is determined from photoconductance measurements together with the measured dopant profiles via numerical simulation, using the latest physical models. These values are compared with calculations based on the interface properties determined from capacitance-voltage and conductance measurements. It is found that the fundamental surface recombination velocity of electrons, $S_{n 0}$, which describes the chemical passivation of the interface, is independent of the surface boron concentration $N_{s}$ for $N_{s} \leq 3 \times 10^{19} \mathrm{~cm}^{-3}$, and in excellent agreement with values calculated from the interface state density $D_{i t}$ and capture coefficients $c_{n}$ and $c_{p}$ measured on undiffused boron-doped surfaces. We conclude that the physical properties of the $\mathrm{Si}-\mathrm{Al}_{2} \mathrm{O}_{3}$ interface are independent of the boron dopant concentration over this range. () 2014 AIP Publishing LLC. [http://dx.doi.org/10.1063/1.4867643]
\end{abstract}

\section{INTRODUCTION}

Effective electrical passivation of crystalline silicon (c$\mathrm{Si}$ ) surfaces is an essential requirement for achieving high efficiencies in c-Si photovoltaic devices, which continue to make up the great majority of industrial production. In recent years, thin-film amorphous aluminum oxide $\left(\mathrm{Al}_{2} \mathrm{O}_{3}\right)$ has attracted a great deal of interest due to its outstanding properties in this regard. ${ }^{1-7} \mathrm{~A}$ major reason for the interest in $\mathrm{Al}_{2} \mathrm{O}_{3}$ is its ability to very effectively passivate highly doped $p$-type silicon surfaces, which is difficult with conventional silicon nitride $\left(\mathrm{SiN}_{\mathrm{x}}\right)$ passivation layers. ${ }^{8,9}$ This ability is a prerequisite for the fabrication of high-efficiency photovoltaic devices based on $n$-type silicon, with its desirable bulk properties,${ }^{10}$ in which a $p+/ n$ junction is generally formed by boron diffusion.

In order to predict the performance of photovoltaic devices incorporating such films, it is necessary to know the recombination rate at the $\mathrm{Si}-\mathrm{Al}_{2} \mathrm{O}_{3}$ interface. This is usually characterized in terms of an effective surface recombination velocity $S_{\text {eff }}$ with units of $\mathrm{cm} / \mathrm{s} .{ }^{11}$ However, $S_{\text {eff }}$ does not have a single value for a given film, but is a strong function of the surface dopant concentration $N_{s}$, as well as the excess carrier concentration $\Delta n$ when the value of $\Delta n$ is comparable to or greater than $N_{s}$. If $N_{s}$ is fixed, it may be feasible to simply measure $S_{\text {eff }}$ experimentally for the conditions of interest. However, if $N_{s}$ is itself a modeling variable, as is the case if diffusion profiles or bulk wafer doping are to be optimized, the functional dependence $S_{\text {eff }}\left(N_{s}\right)$ must be known.

In principle, this dependence can be calculated from physical theory via the extended (energy dependent) Shockley-Read-Hall equation together with a solution of the

${ }^{\text {a)} E l e c t r o n i c ~ m a i l: ~ l a c h l a n . b l a c k @ a n u . e d u . a u ~}$ surface band-bending problem, given knowledge of the interface state properties and the concentration of these states and of any charge in the dielectric. However, in practice such an approach has previously been subject to large uncertainties. Even for the classical $\mathrm{Si}_{-} \mathrm{SiO}_{2}$ interface-by far the most extensively characterized semiconductor-dielectric system-there remains both a lack of data on minority carrier capture coefficients and considerable variation in the measured majority capture coefficients of the interface states, ${ }^{12}$ some of which may be process-related, and some related to measurement uncertainty. Furthermore, in previous work on the $\mathrm{Si}-\mathrm{SiO}_{2}$ interface, it has been reported or implied that the physical interface parameters relating to recombination are strong functions of $N_{s} \cdot{ }^{13-15}$ The latter greatly complicates any modeling based on these parameters.

Therefore, a common approach for device simulation purposes has been to make use of empirical parameterizations of $S_{\text {eff }}$ derived from measurements of multiple samples with $N_{s}$ spanning the range of interest. ${ }^{16-22}$ However, this approach has serious drawbacks. It limits knowledge of $S_{\text {eff }}$ to the range of $N_{s}$ accessible by experiment and requires that new parameterizations be derived whenever the properties of the passivation layer are changed, which entails extensive tedious experimental work. More importantly, it provides no physical insight into the doping dependence of $S_{\text {eff. }}$.

While a number of authors have reported measurements of $\mathrm{Si}-\mathrm{Al}_{2} \mathrm{O}_{3}$ surface passivation on boron-diffused surfaces spanning a range of $N_{s},{ }^{23-28}$ the interest of these authors has primarily been to demonstrate the effectiveness of the passivation on such surfaces, rather than to gain a quantitative physical understanding of the passivation mechanisms.

In this paper, we determine $S_{\text {eff }}$ as a function of $N_{s}$ for $\mathrm{Al}_{2} \mathrm{O}_{3}$-passivated boron-doped surfaces via two independent methods: (1) from photoconductance-based measurements of 
carrier recombination on diffused and undiffused substrates with a range of $N_{s}$, and (2) from measurements of the $\mathrm{Si}-\mathrm{Al}_{2} \mathrm{O}_{3}$ interface state density $D_{i t}$ and insulator fixed charge $Q_{f}$ for metal-insulator-semiconductor (MIS) structures at a single moderate dopant density. We show that the latter approach, in conjunction with recently measured values of the $\mathrm{Si}-\mathrm{Al}_{2} \mathrm{O}_{3}$ interface state properties, ${ }^{29}$ permits very accurate quantitative prediction of $S_{\text {eff }}$ over a wide range of $N_{s}$. Furthermore, we demonstrate that the fundamental surface recombination velocity of electrons, $S_{n 0}$, which is the energy-integrated product of $D_{i t}$ and the electron capture coefficient, $c_{n}$, is independent of the surface boron concentration within the examined range.

We conclude, therefore, that unlike what has been reported for $\mathrm{Si}-\mathrm{SiO}_{2}$, the $\mathrm{Si}-\mathrm{Al}_{2} \mathrm{O}_{3}$ interface properties are independent of the surface boron concentration. For a given set of deposition and annealing conditions, it is therefore possible to predict the recombination rate at any boron-doped silicon surface (diffused or undiffused) passivated with $\mathrm{Al}_{2} \mathrm{O}_{3}$ by using the $\mathrm{Si}-\mathrm{Al}_{2} \mathrm{O}_{3}$ interface properties measured on a moderately doped $\mathrm{Si}$ wafer. The combination of $S_{n O}$ and $Q_{f}$ is shown to provide an efficient doping and injection-independent parameterisation of the passivation properties of this interface, superior to $S_{\text {eff. }}$.

\section{SAMPLE PREPARATION}

To provide samples with a range of high $N_{s}$, borondiffused symmetrical $p+/ p / p+$ structures were prepared on high resistivity ( $>100 \Omega \mathrm{cm}$ ) $p$-type (B-doped), $\langle 100\rangle$, floatzone silicon substrates. These received a surface-damage etch in an HF: $\mathrm{HNO}_{3}$ solution, followed by a standard RCA clean and dilute HF dip. Boron diffusions were performed in a quartz tube furnace from a $\mathrm{BBr}_{3}$ source. Three separate diffusions with different deposition temperatures were performed on different samples. The diffusion parameters and resulting sheet resistances measured by four-point probe are given in Table I.

After the diffusion, the wafers were stripped of borosilicate glass by immersion in dilute HF until hydrophobic. They were then RCA cleaned and subjected to different drive-in anneals in an $\mathrm{N}_{2}$ ambient at $1100^{\circ} \mathrm{C}$ for either 30 ,
90 , or $180 \mathrm{~min}$. The drive-in step redistributes the diffused boron into the wafer, resulting in a stretch-out of the dopant profile and providing a broad range of $N_{s}$. One sample from each of the three diffusions did not receive a drive-in. The resulting dopant profiles were measured by a WEP Wafer Profiler CVP21 tool using the electrochemical capacitance-voltage (ECV) method. The surface concentrations were extrapolated from quadratic fits of the near-surface data.

Fig. 1 shows the resulting measured dopant concentration profiles, while Table I summarizes their key properties. The different diffusion and drive-in steps resulted in a wide spread in surface concentration and profile depth, with $N_{s}$ ranging from $4.0 \times 10^{18}$ to $5.2 \times 10^{19} \mathrm{~cm}^{-3}$. For the samples which did not receive a drive-in, the profile peak occurs away from the surface due to the oxide acting as a sink for near-surface boron during the oxidation stage of the diffusion. Because of the resulting steep near-surface profiles, there is consequently somewhat greater uncertainty in the extrapolated values of $N_{s}$ for these samples.

Following the diffusion, drive-in, and an RCA clean, the samples were passivated with $\mathrm{Al}_{2} \mathrm{O}_{3}$ and characterized. Two deposition methods were sequentially examined: atmospheric pressure chemical vapor deposition (APCVD) and thermal atomic layer deposition (ALD). Samples were first passivated by APCVD $\mathrm{Al}_{2} \mathrm{O}_{3}$, then this layer was removed in $\mathrm{HF}$, and the samples were again RCA cleaned and repassivated with $\mathrm{Al}_{2} \mathrm{O}_{3}$ using thermal ALD. The APCVD films were characterized both in the as-deposited and annealed state, while the ALD films are known to be unstable as-deposited, and were therefore annealed immediately after deposition.

The APCVD $\mathrm{Al}_{2} \mathrm{O}_{3}$ films were deposited using an APCVD belt furnace system (Schmid Thermal Systems) ${ }^{30}$ from triethyldialuminium-tri-(sec-butoxide) (TEDA-TSB) and water vapor at a substrate temperature of $\sim 440{ }^{\circ} \mathrm{C}$ and a $\mathrm{H}_{2} \mathrm{O}$ :TEDA-TSB molar ratio of $\sim 9: 1$. The thickness of the films was $\sim 12 \mathrm{~nm}$, as determined by spectral reflectance measurements on co-deposited polished samples using the refractive index of Ref. 6. Thermal ALD $\mathrm{Al}_{2} \mathrm{O}_{3}$ films were deposited in a Beneq TFS 200 reactor from trimethylaluminium (TMA) and water vapor at a substrate temperature of $200^{\circ} \mathrm{C}$. These had a thickness of $20 \mathrm{~nm}$. Annealing of both

TABLE I. Properties of the boron diffusion profiles prepared in this work. Depth refers to the distance from the surface at which the boron concentration is equal to $10^{17} \mathrm{~cm}^{-3}$.

Diffusion temp. $\left({ }^{\circ} \mathrm{C}\right) \quad$ Drive-in time (min) $\quad$ Sheet resistance $(\Omega / \square) \quad$ Surface boron concentration $\left(\mathrm{cm}^{-3}\right) \quad$ Peak boron concentration $\left(\mathrm{cm}^{-3}\right) \quad$ Depth $(\mu \mathrm{m})$

\begin{tabular}{|c|c|c|c|c|c|}
\hline \multirow[t]{4}{*}{1000} & 0 & 26.75 & $2.8 \times 10^{19}$ & $1.1 \times 10^{20}$ & 0.92 \\
\hline & 30 & 27.5 & $5.2 \times 10^{19}$ & $5.4 \times 10^{19}$ & 1.67 \\
\hline & 90 & 29.5 & $3.3 \times 10^{19}$ & $3.3 \times 10^{19}$ & 2.27 \\
\hline & 180 & 31.25 & $2.5 \times 10^{19}$ & $2.5 \times 10^{19}$ & 2.75 \\
\hline \multirow[t]{4}{*}{950} & 0 & 74.25 & $2.5 \times 10^{19}$ & $4.7 \times 10^{19}$ & 0.46 \\
\hline & 30 & 69.75 & $2.3 \times 10^{19}$ & $2.3 \times 10^{19}$ & 1.24 \\
\hline & 90 & 70.0 & $1.5 \times 10^{19}$ & $1.5 \times 10^{19}$ & 1.76 \\
\hline & 180 & 69.0 & $1.2 \times 10^{19}$ & $1.2 \times 10^{19}$ & 2.27 \\
\hline \multirow[t]{4}{*}{900} & 0 & 186.75 & $7.0 \times 10^{18}$ & $1.8 \times 10^{19}$ & 0.36 \\
\hline & 30 & 158.5 & $7.5 \times 10^{18}$ & $7.5 \times 10^{18}$ & 0.98 \\
\hline & 90 & 146.0 & $5.5 \times 10^{18}$ & $5.5 \times 10^{18}$ & 1.37 \\
\hline & 180 & 139.5 & $4.0 \times 10^{18}$ & $4.0 \times 10^{18}$ & 1.77 \\
\hline
\end{tabular}




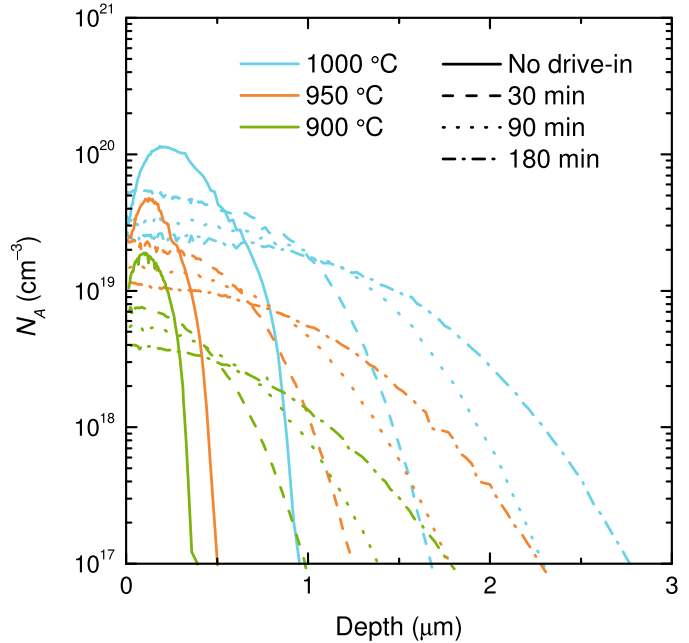

FIG. 1. Acceptor concentration profiles measured by the ECV method for the boron diffusions used in this work. Three different diffusion temperatures and three different post-diffusion drive-in annealing times were used to achieve a broad range of surface concentrations.

APCVD and ALD films was performed in a quartz tube furnace at $425^{\circ} \mathrm{C}$ for $30 \mathrm{~min}$ in an $\mathrm{N}_{2}$ ambient.

In order to extend the range of examined $N_{s}$, additional lifetime samples were prepared on various undiffused, boron-doped $\langle 100\rangle$ float-zone silicon substrates with bulk resistivity ranging from 1.6 to $0.5 \Omega \mathrm{cm}$, corresponding to boron concentrations of $0.9-3.3 \times 10^{16} \mathrm{~cm}^{-3}$. Like the diffused samples, these received a surface-damage etch in $\mathrm{HF}: \mathrm{HNO}_{3}$, followed by an RCA clean and HF dip before $\mathrm{Al}_{2} \mathrm{O}_{3}$ deposition.

\section{PHOTOCONDUCTANCE MEASUREMENTS}

\section{A. Experimental details and results}

The effective excess carrier lifetime $\tau_{\text {eff }}$ of the symmetrically passivated lifetime structures, both diffused and undiffused, was measured as a function of the average bulk excess carrier density $\Delta n$ using a Sinton Instruments WCT-120 photoconductance tool.

For the undiffused samples, a short flash illumination was used and $\tau_{\text {eff }}$ vs $\Delta n$ was extracted from the resulting photoconductance decay. For these samples, $S_{\text {eff }}$ was calculated directly from the measured value of $\tau_{\text {eff }}$ at $\Delta n=10^{15} \mathrm{~cm}^{-3}$, according to

$$
S_{e f f}=\frac{W}{2}\left(\frac{1}{\tau_{e f f}}-\frac{1}{\tau_{\text {bulk,intrinsic }}}\right),
$$

where $W$ is the wafer thickness and $\tau_{\text {bulk,intrinsic }}$ is the Augerlimited intrinsic bulk lifetime, calculated using the empirical parameterization of Ref. 31. It was therefore assumed that Shockley-Read-Hall recombination in the bulk of the wafers was negligible. For the most lightly doped samples $\left(N_{s}=9.2 \times 10^{15} \mathrm{~cm}^{-3}\right)$, the bulk lifetime was observed to be significantly degraded due to iron contamination, rendering (1) invalid. Therefore for these samples, $S_{\text {eff }}$ was instead extracted from the slope of the inverse lifetime in high injection via (A7) and (2), where the lifetime was measured after strong illumination to dissociate the iron-boron pairs.
Photoconductance measurements of the diffused samples were performed using quasi-steady-state illumination with the generalized analysis. ${ }^{32}$ The optical constant required to calculate the generation rate was determined from reflectance and transmission measurements of the passivated samples using a Perkin-Elmer Lambda 1050 spectrophotometer, together with the measured lamp spectrum and quantum efficiency of the reference cell, as described in Ref. 33. It was confirmed that the use of this optical constant resulted in good agreement (within $\pm 2 \%$ ) between lifetime values determined from the illuminated part of the measurement before the cutoff of the flash, and those determined from the unilluminated transient tail measured after the cutoff.

For the diffused samples, the recombination current density $J_{0}$ was extracted from $\tau_{e f f}(\Delta n) \mathrm{via}^{34}$

$$
J_{0}=\frac{q W n_{i}^{2}}{2} \frac{d}{d \Delta n}\left(\frac{1}{\tau_{e f f}}-\frac{1}{\tau_{\text {bulk,intrinsic }}}\right),
$$

where $q$ is the fundamental charge, and $n_{i}=9.7 \times 10^{9} \mathrm{~cm}^{-3}$ is the intrinsic carrier concentration in silicon, calculated using the expression of Ref. 35 at $300 \mathrm{~K}$. Note that the measured quantity in this case is, in fact, $J_{0} / n_{i}^{2}$, which is essentially independent of temperature and is the value relevant to recombination, and the choice of $n_{i}$ used to extract $J_{0}$ is arbitrary. We present the results in terms of $J_{0}$ rather than $J_{0} / n_{i}^{2}$ only because this is the convention. It is however a trivial matter to apply a different value of $n_{i}$ in order to make the $J_{0}$ values comparable with values quoted by other authors using different values of $n_{i}$. The value of the derivative in (2) was extracted using a linear fit over the range of $\Delta n=8-12 \times 10^{15} \mathrm{~cm}^{-3}$. This range was chosen as that for which the local value of the derivative showed the least variation with $\Delta n$ for all samples.

Fig. 2 plots the resulting $J_{O}$ values as a function of $N_{s}$. It also gives ranges for the sheet resistance of the diffusions. The figure shows that $J_{0}$ increases roughly monotonically with $N_{s}$ for each type of $\mathrm{Al}_{2} \mathrm{O}_{3}$. This trend is caused by an

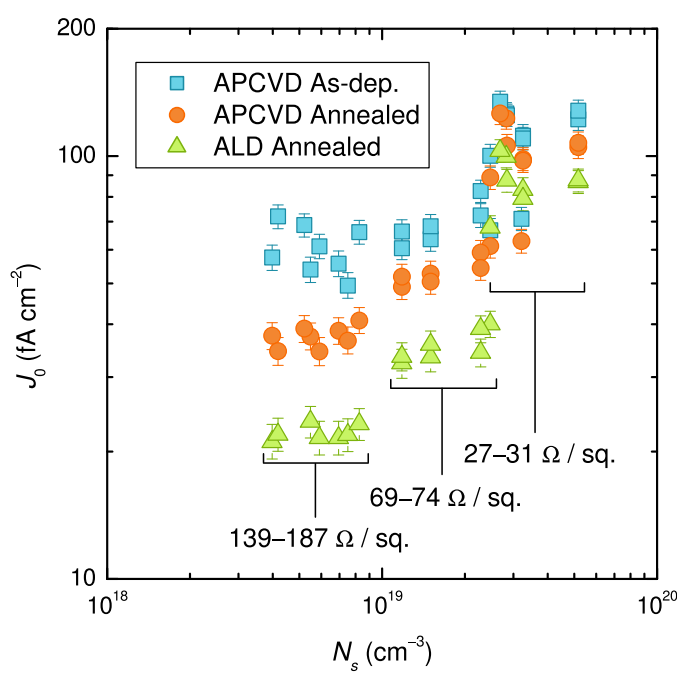

FIG. 2. Measured recombination current density $J_{0}$ vs. surface boron concentration $N_{S}$ for the diffusion profiles of Fig. 1 passivated with $\mathrm{Al}_{2} \mathrm{O}_{3}$ deposited either by APCVD (as-deposited and annealed), and thermal ALD (annealed only). Error bars show uncertainty due to error in the substrate thickness and the optical constant. 
increase in Auger recombination as the silicon becomes more heavily doped. The samples that did not receive a drive-in lie on a slightly different trend because their peak dopant concentration is greater than $N_{s}$.

Fig. 2 also shows that the as-deposited APCVD samples have the highest $J_{0}$ and therefore provide the poorest passivation. The $J_{0}$ of these APCVD samples decreases once they are annealed, and their magnitudes correspond to very good surface passivation. For example, for the lightest B diffusion, the $J_{0}$ is just $35 \mathrm{fA} / \mathrm{cm}^{2}$. This level of passivation is of particular interest to solar cell manufacturers due to the low-cost nature of APCVD. ${ }^{30}$ The $J_{0}$ of annealed thermal ALD samples is lower still with a $J_{O}$ of $20 \mathrm{fA} / \mathrm{cm}^{2}$ on the lightest B diffusions. The $J_{0}$ values for these samples are in good agreement with the few previously reported passivation results for $\mathrm{Al}_{2} \mathrm{O}_{3}$ from thermal $\mathrm{ALD}$ on boron-diffused surfaces, ${ }^{36,37}$ despite significantly different post-deposition thermal processing. Even lower values of $J_{0}$, down to less than $10 \mathrm{fA} / \mathrm{cm}^{2}$, have been reported for $\mathrm{Al}_{2} \mathrm{O}_{3}$ from plasma-assisted ALD and plasma-enhanced chemical vapour deposition (PECVD). ${ }^{23-25,27,28,36}$

\section{B. Determination of $S_{\text {eff }}$ from $J_{0}$ data}

The $J_{0}$ extracted via (2) includes contributions from Auger recombination in the heavily doped $p+$ region and Shockley-Read-Hall recombination through interface states at the silicon surface. To evaluate the surface passivation provided by the $\mathrm{Al}_{2} \mathrm{O}_{3}$, it is therefore necessary to extract the contribution due to surface recombination. This extraction is performed by numerical simulation of the measured dopant profiles, as described in Ref. 38, to determine the surface recombination velocity $S_{\text {eff }}$, which then allows a comparison of the passivation to that attained on undiffused samples.

For such highly doped semiconductor regions, dopinginduced bandgap narrowing becomes significant and must be included in the analysis. For this purpose we used the recent empirical parameterization of Ref. 39, which is based on a large self-consistent dataset, and makes use of the most upto-date values for the physical parameters. We use the Fermi-Dirac variant of this parameterization together with Fermi-Dirac carrier statistics. While this parameterization was derived for phosphorus rather than boron-doped silicon, previous work has shown that the bandgap narrowing data for both dopants agrees quite closely. ${ }^{40}$

Auger recombination in the diffused region was again calculated using the model of Richter et al. ${ }^{31}$ This model gives slightly lower values for the Auger recombination at high dopant concentrations than the widely used model of Dziewior and Schmid, ${ }^{41}$ and substantially lower than that of Kerr and Cuevas, ${ }^{42}$ which was not optimized to fit the data at higher dopant concentrations. It is likely that the use of the model of Ref. 42 in the analysis of some previous measurements of $\mathrm{Al}_{2} \mathrm{O}_{3}$-passivated boron-diffused surfaces ${ }^{25}$ resulted in a significant underestimation of the extracted $S_{\text {eff }}$.

Figs. 3 and 4 plot $S_{\text {eff }}$ against $N_{s}$, where the symbols show the results of the photoconductance measurements, and the lines show the results of simulations based on experimental measurements of the interface properties (as described

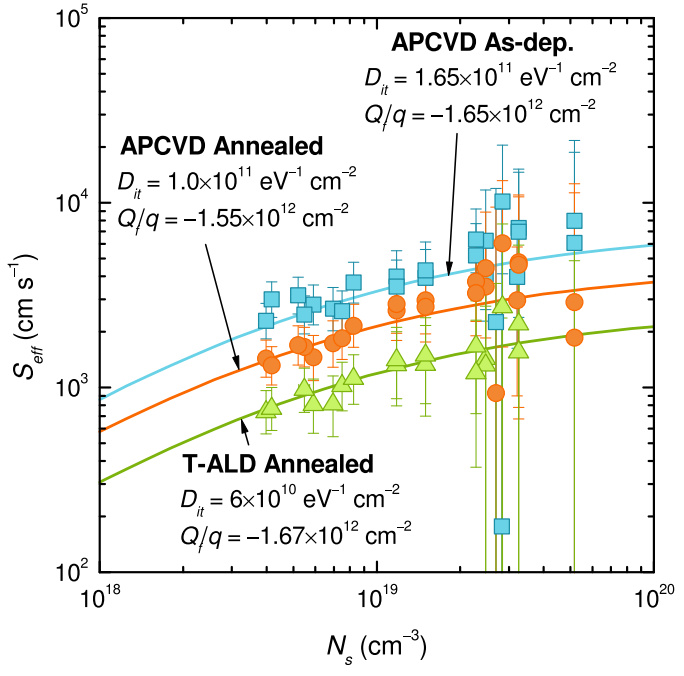

FIG. 3. Effective surface recombination velocity $S_{\text {eff }}$ as a function of surface boron concentration $N_{s}$, extracted from the data of Figs. 1 and 2. Lines show values calculated from the measured interface state density and fixed charge, using the interface state model of Ref. 29.

below). Fig. 3 contains just the diffused samples, and Fig. 4 contains both the diffused and undiffused samples. There is a clear trend of increasing $S_{\text {eff }}$ with increasing $N_{s}$ in all cases.

Note that the same samples shown in Fig. 3 were also passivated with APCVD films deposited at a slightly higher deposition temperature $\left(\sim 490^{\circ} \mathrm{C}\right)$ (data not shown). In this case, they exhibited $S_{\text {eff }}$ values very similar to those of the annealed APCVD data shown, both as-deposited and after annealing. This is consistent with our previous observation that at higher deposition temperatures both $D_{i t}$ and $Q_{f}$ saturate and are not significantly affected by post-deposition annealing. ${ }^{6}$ The data are not included here to avoid cluttering the figure.

Error bars for the diffused samples in Figs. 3 and 4 show the combined error due to the stated uncertainty in the

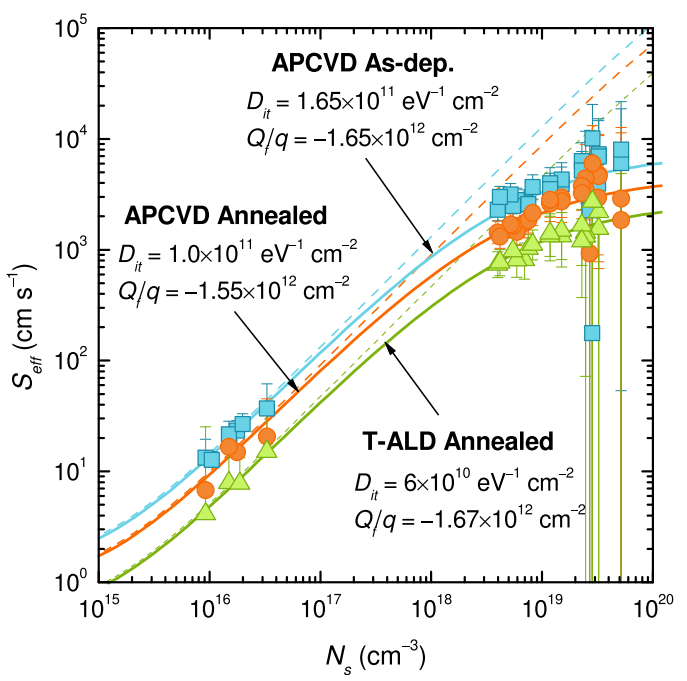

FIG. 4. The data of Fig. 3 replotted on a broader scale, with the addition of $S_{\text {eff }}$ data measured on more lightly doped undiffused surfaces. As in Fig. 3, solid lines show predicted values calculated from the measured interface properties. Dashed lines show the predictions of an approximate model in which the surface majority carrier concentration is proportional to $Q_{f}^{2}$. This model is a good approximation for undiffused surfaces when $Q_{f}$ is large. 
bandgap narrowing parameterization of Ref. 39, together with the error in $J_{0}$ shown in Fig. 2. For the samples with $N_{s}>2 \times 10^{19} \mathrm{~cm}^{-3}$ there is large scatter in the data. For such heavily doped surfaces, Auger recombination in the diffused region dominates $J_{0}$, making the extraction of $S_{\text {eff }}$ subject to significant relative uncertainty. This occurs both because Auger recombination increases with higher doping, and also because surface recombination is reduced due to suppression of the surface minority carrier concentration at higher $N_{s}$. This uncertainty is compounded by uncertainty in the minority carrier concentration due to the onset of significant bandgap narrowing, and is reflected in the significantly larger error bars for this data. Note that the assumption of higher bandgap narrowing corresponds to lower extracted $S_{\text {eff }}$, and vice versa. Error bars for the undiffused samples show the upper limit of $S_{\text {eff }}$ assuming an infinite bulk lifetime.

The clear increase in $S_{\text {eff }}$ with $N_{s}$ misleadingly suggests that the physical interface properties themselves are changing with doping. This might, for example, have been caused by a decrease in negative charge $Q_{f}$ or an increase of interface states $D_{i t}$. It is next shown, however, that neither $Q_{f}$ nor $D_{i t}$ depends on $N_{s}$, and that instead, the increase of $S_{\text {eff }}$ with $N_{s}$ is simply a consequence of the definition of $S_{\text {eff }}$.

\section{CAPACITANCE-VOLTAGE MEASUREMENTS}

\section{A. Experimental details and results}

Conventional $\mathrm{C}-\mathrm{V}$ measurements of $D_{i t}$ are difficult on highly doped surfaces because the interface state capacitance appears in parallel with a very large substrate capacitance, making it challenging to extract $D_{i t}$ accurately, especially when $D_{i t}$ is small. The large voltages required to drive the surface from inversion to accumulation can also lead to dielectric breakdown, making low frequency measurements impossible due to high leakage currents through the film. $\mathrm{C}-\mathrm{V}$ measurements were therefore performed only on moderately doped undiffused substrates, and $S_{\text {eff }}\left(N_{s}\right)$ was calculated assuming that the measured values of $D_{i t}$ and $Q_{f}$ were independent of $N_{s}$. This assumption is subsequently justified by comparison with $S_{\text {eff }}$ determined from the photoconductance measurements.

The metal-insulator-semiconductor structures for $\mathrm{C}-\mathrm{V}$ measurements were prepared on $2.7 \Omega \cdot \mathrm{cm} \quad\left(N_{A}=5.2\right.$ $\times 10^{15} \mathrm{~cm}^{-3}$ ) boron-doped $\langle 100\rangle$ polished $\mathrm{Si}$ substrates, which received an RCA clean and $\mathrm{HF}$ dip prior to $\mathrm{Al}_{2} \mathrm{O}_{3}$ deposition. Gate contacts were formed by thermal evaporation of Al through a shadow mask, while GaIn paste was used to make an ohmic contact at the rear. High frequency (1 $\mathrm{MHz}$ ) and quasi-static $\mathrm{C}-\mathrm{V}$ measurements were performed at multiple points on each sample using an HP 4284 A precision LCR meter and HP 4140B picoammeter/DC voltage source, with analysis as described in Ref. 29. Representative extracted $D_{i t}(E)$ profiles for each of the films are presented in Fig. 5, while averaged extracted values of $Q_{f}$ and $D_{i t}$ at midgap are presented in Table II. All three films have similar $Q_{f}$, being distinguished principally by differences in $D_{i t}$.

Note that while the $\mathrm{C}-\mathrm{V}$ data for the APCVD films was measured using the standard voltage sweep from inversion to accumulation, the data for the ALD films was measured

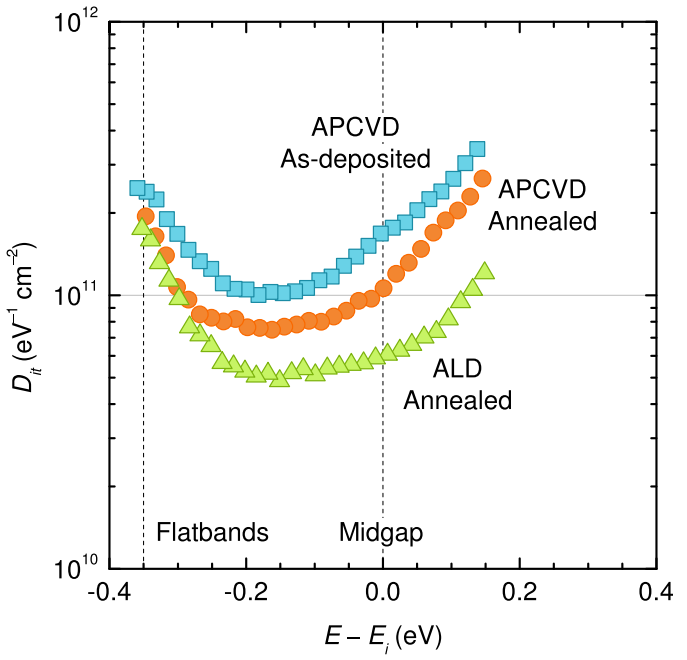

FIG. 5. Interface state density $D_{i t}$ as a function of energy $E$ with respect to midgap, determined from capacitance-voltage measurements for each of the $\mathrm{Al}_{2} \mathrm{O}_{3}$ films. Note that the values shown here are taken from representative single measurements, while the average midgap $D_{i t}$ values in Table II are taken from multiple such measurements.

using a voltage sweep in the opposite direction. This is because it was found that for these films the apparent $D_{i t}$ showed a substantial dependence on the sweep direction, with a voltage sweep in the standard direction resulting in an apparent $D_{i t}$ that was substantially larger than that of even the as-deposited APCVD films. As it cannot be the case that the recombination-active interface state density is greater for the ALD films, we suspect that these additional apparent interface states represent recombination-ineffective charge states, perhaps slow traps associated with the slight hysteresis in the $\mathrm{C}-\mathrm{V}$ characteristics also observed for these films but which is not present for the APCVD films. Hence, for the ALD films, we use the $\mathrm{C}-\mathrm{V}$ data resulting from the accumulation to inversion voltage sweep, which results in a lower and more plausible value for $D_{i t}$. Care was taken to use sufficiently slow $(0.01$ and $0.02 \mathrm{~V} / \mathrm{s})$ voltage sweeps, such that thermal equilibrium would be maintained through the midgap region for this measurement.

\section{B. Determination of $S_{\text {eff }}$ from C-V data}

The negative $Q_{f}$ of the $\mathrm{Al}_{2} \mathrm{O}_{3}$ films (Table II) results in accumulation of majority carriers at the p-type surfaces under consideration. Under these conditions (and when not very close to thermal equilibrium), $S_{\text {eff }}$ is related to $D_{i t}$ and $Q_{f}$ by (see Appendix),

TABLE II. $\mathrm{Si}-\mathrm{Al}_{2} \mathrm{O}_{3}$ interface properties determined by $\mathrm{C}-\mathrm{V}$ measurements: fixed insulator charge $Q_{f}$, midgap interface state density $D_{i t}$, and corresponding fundamental surface recombination velocity of electrons, $S_{n 0}$, calculated from (6).

\begin{tabular}{lccc}
\hline \hline & $\begin{array}{c}Q_{f} / q \\
\left(10^{12} \mathrm{~cm}^{-2}\right)\end{array}$ & $\begin{array}{c}\text { Midgap } \\
D_{i t}\left(10^{11} \mathrm{~cm}^{-2} \mathrm{eV}^{-1}\right)\end{array}$ & $\begin{array}{c}S_{n 0} \\
\left(10^{3} \mathrm{~cm} / \mathrm{s}\right)\end{array}$ \\
\hline Passivation layer & -1.65 & 1.65 & 10.7 \\
APCVD As-dep. & -1.55 & 1.00 & 6.5 \\
T-ALD annealed & -1.67 & 0.60 & 3.9 \\
\hline \hline
\end{tabular}




$$
S_{e f f}=S_{n 0} \frac{n_{s}}{n_{d}}
$$

where

$$
S_{n 0}=\int_{E_{V}}^{E_{C}} D_{i t} c_{n} d E
$$

$S_{n 0}$ is the fundamental surface recombination velocity of electrons, ${ }^{43}$ and $n_{s} / n_{d}$ is the ratio of the electron concentration at the surface to that at the edge of the quasi-neutral region in the semiconductor. This ratio is a function of $Q_{f}$, and generally must be calculated numerically.

The measured midgap interface state density can be used in conjunction with the experimentally based $\mathrm{Si}-\mathrm{Al}_{2} \mathrm{O}_{3}$ interface state model of Ref. 29 to calculate $S_{n 0}$ via (4). In the model of Ref. 29, which was derived from measurements of the frequency-dependent parallel conductance of the $\mathrm{Si}-\mathrm{Al}_{2} \mathrm{O}_{3}$ interface states, the dominant recombination-active defect for surfaces where holes are the majority carrier is described by a Gaussian distribution of states centered just below midgap with energy-independent $c_{n}$ and $c_{p}$. The integral of this distribution with respect to energy is easily calculated to be

$$
\int_{E_{V}}^{E_{C}} D_{i t} d E=0.18 \times D_{i t, \text { midgap }},
$$

expressed in terms of $D_{i t}$ measured at midgap. Assuming the conditions relating to (4) hold (as they do in our case), $S_{n 0}$ is then given by

$$
S_{n 0}=c_{n} \int_{E_{V}}^{E_{C}} D_{i t} d E=6.5 \times 10^{-8} \times D_{\text {it,midgap }},
$$

where $S_{n 0}$ is in units of $\mathrm{cm} / \mathrm{s}$ and $D_{\text {it,midgap }}$ is in units of $\mathrm{cm}^{-2} \mathrm{eV}^{-1}$. The $S_{n 0}$ values calculated from (6) are summarized in Table II.

The surface electron concentration $n_{s}$ was calculated from the surface dopant density $N_{s}$ and insulator fixed charge $Q_{f}$ of Table II using Fermi-Dirac statistics together with an iterative solution of the silicon surface potential, in the manner described by Ref. 44. For this purpose, we treat the dopant concentration as constant across the width of the surface charge profile, and equal to the measured $N_{s}$ of the diffused samples. This should be a reasonable approximation, even for the steeper dopant profiles, since the width of the surface charge profile in accumulation for such high $N_{s}$ is only on the order of $10 \mathrm{~nm}$ or less.

$S_{\text {eff }}\left(N_{s}\right)$ calculated from the measured $D_{i t}$ and $Q_{f}$ via (3)-(6) for each of the passivation layers are shown as solid curves in Figs. 3 and 4. These lines agree very well-both in terms of trends and absolute values - with the symbols that were independently determined from the photoconductance measurements. The agreement is remarkable considering that both $S_{\text {eff }}$ and $N_{s}$ span several orders of magnitude. The dashed lines in Fig. 4 show $S_{\text {eff }}$ calculated using the approximate analytical expression of (A7) (see Appendix), in which the surface majority carrier concentration is assumed proportional to $Q_{f}^{2}$. While this expression is unsuitable for highly doped surfaces, it is a good approximation for most undiffused surfaces when $Q_{f}$ is large, as in this case.

The calculation of $S_{\text {eff }}$ from the measured interface properties (i.e., from the $\mathrm{C}-\mathrm{V}$ measurements on undiffused $\mathrm{Si}$ ) was predicated on the assumption that these properties were independent of the surface boron concentration $N_{s}$. The excellent agreement in $S_{\text {eff }}$ over such a wide range of $N_{s}$ is strong evidence that this assumption is indeed valid.

\section{DETERMINATION OF $\boldsymbol{S}_{\boldsymbol{n}}$}

The large variation of $S_{\text {eff }}$ with $N_{s}$ highlights the limitations of this parameter as a means of describing surface passivation, as discussed in Ref. 11. It is useful to present the surface recombination rate in terms of $S_{\text {eff }}$ because this parameter is easily determined and it includes the influence of both charge-assisted and chemical surface passivation. It is also often used to describe surface recombination and is hence familiar to many readers. However, because $S_{\text {eff }}$ depends on both $Q_{f}$ and $N_{s}$, it can only provide a complete description of the interface passivation properties when quoted together with these parameters. It is not very meaningful to directly compare $S_{\text {eff }}$ values measured for substrates with different $N_{s}$, as is commonly done.

In contrast, $Q_{f}$ and $S_{n 0}$ or $S_{p 0}$ in combination constitute a complete description of the interface properties (so long as the assumptions pertaining to (A2) apply) and do not possess an intrinsic dependence on $N_{s}$. That is, a dependence on $N_{s}$ would only arise via a dependence of the physical properties of the interface $\left(Q_{f}, D_{i t}, c_{n}\right.$, and/or $\left.c_{p}\right)$ on the dopant concentration.

To reinforce this point, it is worthwhile to analyze the data of Figs. 3 and 4 to extract $S_{n 0}$, using the relation defined in (3). This decouples the effect of $Q_{f}$ from $S_{\text {eff }}$, leaving a term which depends only on the concentration of interface states and their effectiveness as recombination centers. Fig. 6 presents the resulting $S_{n 0}$ values, with lines of constant $S_{n 0}$ indicating the values calculated from the measured $D_{i t}$ of Table II via (6). It is clear that, despite experimental scatter, the data are indeed well described by a constant value of $S_{n 0}$,

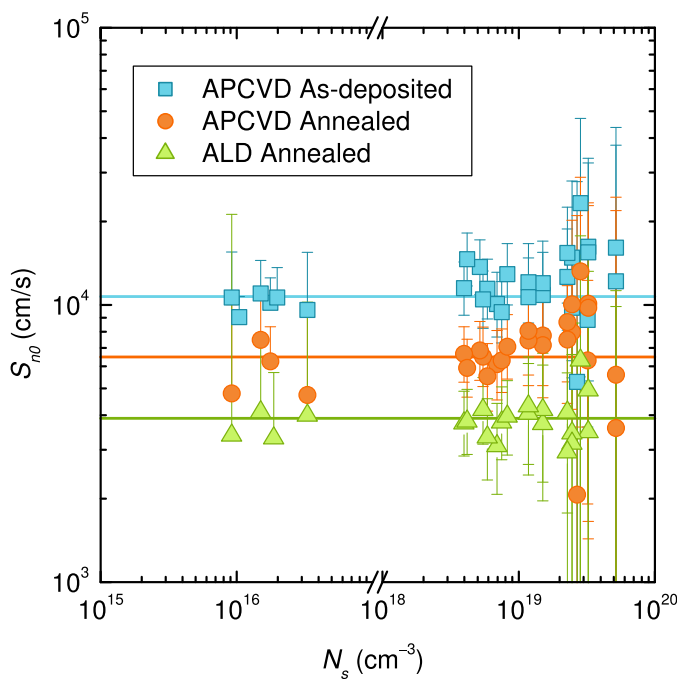

FIG. 6. The fundamental surface recombination velocity of electrons, $S_{n 0}$, extracted from the data of Fig. 4 via (3). Lines show $S_{n 0}$ calculated from the measured interface state density $D_{i t}$ via (6). 
highlighting the advantage of the use of this parameter (or $S_{p 0}$ ) in conjunction with $Q_{f}$ as a means to describe the effectiveness of a given surface passivation. This conclusion holds at least up to $N_{s}=\sim 3 \times 10^{19} \mathrm{~cm}^{-3}$, above which point the experimental uncertainty becomes too large to draw any reliable conclusions, although the lines of constant $S_{n 0}$ remain within the error bounds for all the data.

The uncertainty in $S_{n 0}$ at high $N_{s}$ derives from the increasing importance and eventual dominance of Auger recombination in the heavily doped region, relative to surface recombination. At high $N_{s}$, any error in the Auger recombination model would contribute significantly to error in the extraction of $S_{\text {eff }}$ and $S_{n o}$. This is compounded by uncertainty in the bandgap narrowing model, and hence in the minority carrier concentration, both in the bulk and at the surface. The qualitative effect of using a different bandgap narrowing model in the extraction of $S_{\text {eff }}$, and hence $S_{n 0}$, can be seen in the extent of the error bars for the diffused samples. For $N_{s}<\sim 2-3 \times 10^{19} \mathrm{~cm}^{-3}$, where Auger recombination is less important, the conclusion that $S_{n 0}$ is independent of $N_{s}$ is relatively insensitive to changes in bandgap narrowing, though the agreement with the data of the undiffused samples at lower $N_{s}$ is affected. At higher $N_{s}$, the slope of $S_{n 0}$ vs $N_{S}$ becomes very sensitive to the bandgap narrowing and Auger models. If significantly lower bandgap narrowing were assumed, the extracted $S_{n 0}$ would increase strongly with $N_{s}$ above this point, while for significantly higher bandgap narrowing the extracted $S_{n 0}$ would decrease sharply and even assume (unphysical) negative values at high $N_{s}$.

The $S_{n 0}$ values presented in Fig. 6 and Table II are similar to the value of $\sim 1 \times 10^{4} \mathrm{~cm} / \mathrm{s}$ determined by Ma et al. ${ }^{26}$ for PECVD $\mathrm{Al}_{2} \mathrm{O}_{3} / \mathrm{SiN}_{\mathrm{x}}$ stacks on boron-diffused planar surfaces. The agreement is partly coincidental, since $\mathrm{Ma}$ et al. used the Auger model of Kerr and Cuevas, ${ }^{42}$ and also made use of simulated dopant profiles with higher $N_{s}$ than those measured by ECV. These two factors would have respectively acted to decrease and increase the apparent surface recombination velocity, and may have effectively compensated each other in the calculation of $S_{n 0}$. The values are therefore not directly comparable, but nevertheless they are clearly of the same order of magnitude.

The fact that $S_{n 0}$ appears to be constant for the examined passivation layers could conceivably be exploited in order to provide improved determinations of the other important processes occurring at high dopant concentrations: Auger recombination and bandgap narrowing. A constant $S_{\text {eff }}$ (equal to the thermal velocity) is already often assumed in the extraction of bandgap narrowing from measurements of $J_{0}$ on metal-coated diffused surfaces. ${ }^{17,18,39}$ The ability to make a similar assumption for passivated surfaces could effectively provide multiple coupled equations for a given diffusion profile, possibly even allowing both Auger recombination and bandgap narrowing to be determined simultaneously from the same dataset. The practicality of such a procedure would of course depend on the repeatability of the passivation process.

\section{CONCLUSIONS}

We conclude that, in contrast to what has been reported for thermal $\mathrm{SiO}_{2},{ }^{13-15} D_{i t}, c_{n}$, and $Q_{f}$ at the boron-doped
$\mathrm{Si}-\mathrm{Al}_{2} \mathrm{O}_{3}$ interface are independent of the surface boron concentration $N_{s}$ over a range of at least $9 \times 10^{15} \mathrm{~cm}^{-3}$ to $3 \times 10^{19} \mathrm{~cm}^{-3}$. Furthermore, the recombination rate at this interface can be determined with a high degree of accuracy over the same range, given only knowledge of the insulator fixed charge $Q_{f}$ and the fundamental surface recombination velocity of electrons, $S_{n 0}$, measured at any $N_{s}$. The latter can be determined either from the measured interface state density $D_{i t}$ via (6), or from measurements of the excess carrier recombination lifetime via (1) and (3) when $Q_{f}$ is known. The practical implications of this finding for device simulation and modeling are significant, in that it obviates the need to experimentally assess surface recombination as a function of $N_{s}$ whenever the properties of the film are changed, for example as a result of changing deposition conditions. Most importantly, it provides valuable physical insight into the mechanisms of surface passivation.

\section{ACKNOWLEDGMENTS}

The authors would like to thank J. Bullock, W. Liang, S. P. Phang, F. Rougieux, C. Samundsett, and D. Yan for valuable discussions and advice, and for assistance with sample preparation.

\section{APPENDIX: SURFACE RECOMBINATION THEORY}

The net recombination rate $U_{s}$ of mobile charge carriers via interface states at the surface of a semiconductor is well described by the general energy-dependent form of the Shockley-Read-Hall equation, ${ }^{45,46}$ given by

$$
U_{s}=\int_{E_{V}}^{E_{C}} D_{i t} \frac{p_{s} n_{s}-n_{i}^{2}}{c_{p}^{-1}\left(n_{s}+n_{1}\right)+c_{n}^{-1}\left(p_{s}+p_{1}\right)} d E,
$$

where $E_{C}$ and $E_{V}$ are the conduction and valence band energies, $D_{i t}$ is the density of interface states, $p_{s}$ and $n_{s}$ are the surface hole and electron concentrations, $c_{p}$ and $c_{n}$ are the hole and electron capture coefficients, and $p_{1}$ and $n_{1}$ are the equilibrium hole and electron concentrations for Fermi energy equal to $E$. These last terms account for re-emission of trapped carriers and have the effect of reducing the recombination effectiveness of states located close to the band edges. Note that $D_{i t}, c_{p}, c_{n}, n_{1}$, and $p_{1}$ are functions of $E$, though we have omitted this dependence from (A1) for the sake of brevity.

The surface carrier concentrations $p_{s}$ and $n_{s}$ generally vary from their values in the semiconductor bulk due to the influence of charge located in the insulator and in defect states at the semiconductor-insulator interface. For passivated surfaces with a low concentration of interface states, this charge is generally dominated by the insulator fixed charge $Q_{f}$. Given knowledge of $Q_{f}, n_{s}$, and $p_{s}$ can be calculated in the manner of Ref. 44 using either Boltzmann or Fermi-Dirac statistics.

For a strongly $p$-type surface $\left(p_{s} \gg n_{s}\right.$, or more strictly $\left.c_{p} p_{s} \gg c_{n} n_{s}\right)$, surface recombination is limited by the rate of electron capture, and (A1) reduces to 


$$
U_{s}=\frac{p_{s} n_{s}-n_{i}^{2}}{p_{s}} \int_{E_{V}}^{E_{C}} D_{i t} c_{n} d E
$$

This condition holds for all $p$-type surfaces and most undiffused $n$-type surfaces passivated by a negatively charged dielectric such as $\mathrm{Al}_{2} \mathrm{O}_{3}$. Here we have also used the fact that, under these conditions, $p_{1}, n_{1} \ll p_{s}$ for all interface states not located very close to the band edges, so that these terms can be neglected. Finally we can define $S_{n 0}$ as

$$
S_{n 0}=\int_{E_{V}}^{E_{C}} D_{i t} c_{n} d E
$$

As this term contains all the parameters that describe the interface states, and no others, it therefore encapsulates the chemical passivation of the surface.

Because the surface carrier concentrations $p_{s}$ and $n_{s}$ are generally not known, it is common to describe surface recombination in terms of an effective surface recombination velocity $S_{\text {eff }}$, defined with respect to the excess (non-equilibrium) carrier concentration $\Delta n_{d}$ at the edge of the quasineutral region of the semiconductor,

$$
S_{e f f} \equiv \frac{U_{s}}{\Delta n_{d}} .
$$

From (A2)-(A4), $S_{\text {eff }}$ is related to $S_{n 0}$ via

$$
S_{e f f}=S_{n 0} \frac{p_{s} n_{s}-n_{i}^{2}}{p_{s} \Delta n_{d}} \approx S_{n 0} \frac{n_{s}}{\Delta n_{d}},
$$

where the latter expression applies when the surface carrier concentration is far from thermal equilibrium $\left(p_{s} n_{s} \gg n_{i}^{2}\right)$. For surfaces not in inversion, i.e., where the surface minority carriers are the same as those in the near-surface quasi-neutral region, we can make the further substitution $\Delta n_{d} \approx n_{d}$. Note that it is not necessary to include bandgap narrowing in the calculation of $n_{s}$ and $p_{s}$ in (A5), so long as this effect is approximately constant over the free carrier surface charge profile induced by $Q_{f}$.

Fig. 7 illustrates $S_{\text {eff }}$ calculated from (A5) as a function of the surface dopant concentration $N_{s}$, with $Q_{f}$ as a parameter. Several limiting cases and trends are worth noting. (1) For $Q_{f}=0, n_{s}=n_{d} \approx \Delta n_{d}$, and $S_{\text {eff }}$ is simply equal to $S_{n 0}$ (however, note that in this case the assumption of (A2) that $c_{p} p_{s} \gg c_{n} n_{s}$ is unlikely to hold outside of low injection, so that in practice $U_{s}$ will be limited by both $c_{p}$ and $c_{n}$, and $S_{\text {eff }}$ will be less than $S_{n 0}$ unless $N_{s} \gg \Delta n_{d}$ ). (2) For nonnegligible $Q_{f}, S_{\text {eff }}$ is a strong function of $N_{s}$. However, when $N_{s}$ is very large, $p_{s}$ and $n_{s}$ become nearly independent of $Q_{f}$, and $S_{\text {eff }}$ again approaches $S_{n 0}$ regardless of $Q_{f}$.

Making the substitution $p_{s} n_{s} \approx\left(N_{s}+\Delta n_{d}\right) \Delta n_{d}$, (A5) can be rewritten as

$$
S_{e f f} \approx S_{n 0} \frac{\left(N_{s}+\Delta n_{d}\right)}{p_{s}} .
$$

Using the approximate expression for $p_{s}$ when $Q_{f}$ is large, ${ }^{11}$ this becomes

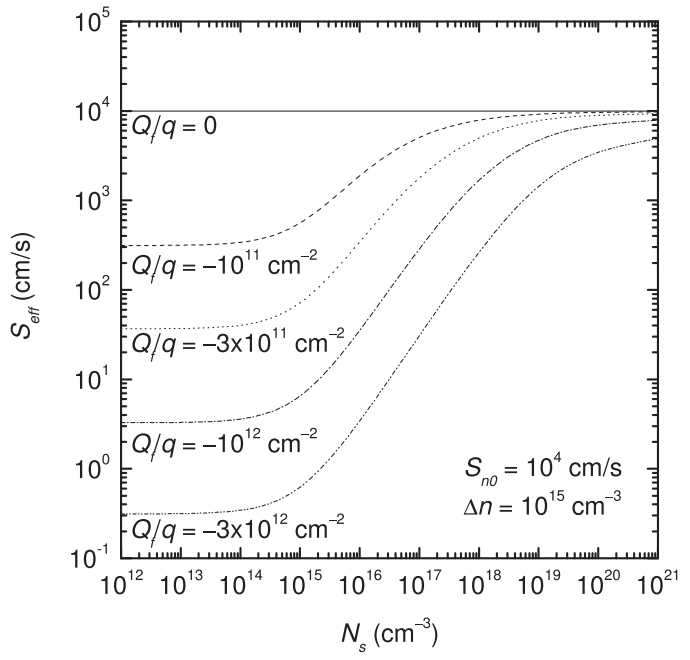

FIG. 7. Effective surface recombination velocity $S_{\text {eff }}$, calculated from (A5) with $S_{n 0}=10^{4} \mathrm{~cm} / \mathrm{s}$ and $\Delta n=10^{15} \mathrm{~cm}^{-3}$, vs. surface acceptor concentration $N_{s}$, with insulator fixed charge $Q_{f}$ as a parameter. As noted in the text, the assumptions relating to (A5) are unlikely to hold in practice for $Q_{f}=0$ (and to a lesser extent for $Q_{f} / q=-10^{11} \mathrm{~cm}^{-3}$ ), so that $S_{\text {eff }}$ will be limited by $c_{p}$ as well as $c_{n}$.

$$
S_{e f f} \approx S_{n 0} \frac{2 \varepsilon_{s} k T}{q^{2} Q_{f}^{2}}\left(N_{s}+\Delta n_{d}\right)=\frac{J_{0}}{q n_{i}^{2}}\left(N_{s}+\Delta n_{d}\right) .
$$

Equation (A7) provides the basis for the extraction of the $J_{O}$ of undiffused surfaces passivated by a charged dielectric in the same way as for diffused surfaces via (2). Under high injection conditions (low $N_{s}$ in Fig. 7), $\Delta n_{d} \gg N_{s}$, and (A6) becomes

$$
S_{e f f} \approx S_{n 0} \frac{\Delta n_{d}}{p_{s}}
$$

i.e., $S_{\text {eff }}$ is independent of $N_{s}$ but dependent on the injection level. Note that analogous expressions to (A2)-(A8) apply for $S_{p 0}$, in the case when electrons rather than holes are the majority carrier at the surface.

${ }^{1}$ G. Agostinelli, A. Delabie, P. Vitanov, Z. Alexieva, H. F. W. Dekkers, S. De Wolf, and G. Beaucarne, Sol. Energy Meter. Sol. Cells 90, 3438 (2006).

${ }^{2}$ B. Hoex, S. B. S. Heil, E. Langereis, M. C. M. van de Sanden, and W. M. M. Kessels, Appl. Phys. Lett. 89, 042112 (2006).

${ }^{3}$ T. T. Li and A. Cuevas, Phys. Status Solidi (RRL) - Rapid Res. Lett. 3, 160 (2009).

${ }^{4}$ P. Saint-Cast, D. Kania, M. Hofmann, J. Benick, J. Rentsch, and R. Preu, Appl. Phys. Lett. 95, 151502 (2009).

${ }^{5}$ S. Miyajima, J. Irikawa, A. Yamada, and M. Konagai, Appl. Phys Express 3, 012301 (2010).

${ }^{6}$ L. E. Black and K. R. McIntosh, Appl. Phys. Lett. 100, 202107 (2012).

${ }^{7}$ X. Zhang, A. Cuevas, and A. Thomson, IEEE J. Photovolt. 3, 183 (2013).

${ }^{8}$ S. Dauwe, L. Mittelstädt, A. Metz, and R. Hezel, Prog. Photovolt.: Res. Appl. 10, 271 (2002).

${ }^{9}$ M. J. Kerr, Ph.D. dissertation (Australian National University, Canberra, 2002).

${ }^{10}$ D. MacDonald and L. J. Geerligs, Appl. Phys. Lett. 85, 4061 (2004).

${ }^{11}$ K. R. McIntosh and L. E. Black, "On effective surface recombination parameters," J. Appl. Phys. (submitted).

${ }^{12}$ A. G. Aberle, S. Glunz, and W. Warta, J. Appl. Phys. 71, 4422 (1992).

${ }^{13}$ J. Snel, Solid-State Electron. 24, 135 (1981).

${ }^{14}$ M. Y. Ghannam, R. P. Mertens, R. F. De Keersmaecker, and R. J. van Overstraeten, IEEE Trans. Electron Devices 32, 1264 (1985). 
${ }^{15} \mathrm{H}$. Jin, W. E. Jellett, Z. Chun, K. J. Weber, A. W. Blakers, and P. J. Smith, Appl. Phys. Lett. 92, 122109 (2008).

${ }^{16}$ R. R. King, R. A. Sinton, and R. M. Swanson, IEEE Trans. Electron Devices 37, 365 (1990).

${ }^{17}$ R. R. King and R. M. Swanson, IEEE Trans. Electron Devices 38, 1399 (1991).

${ }^{18}$ A. Cuevas, P. A. Basore, G. Giroult-Matlakowski, and C. Dubois, J. Appl. Phys. 80, 3370 (1996).

${ }^{19}$ A. Cuevas, M. Stuckings, J. Lau, and M. Petravic, in Proceedings of the $14^{\text {th }}$ European Photovoltaic Solar Energy Conference, Barcelona, 1997 (HS Stephens, Falmerston, UK, 1997), p. 2416.

${ }^{20}$ P. P. Altermatt, J. O. Schumacher, A. Cuevas, M. J. Kerr, S. W. Glunz, R. R. King, G. Heiser, and A. Schenk, J. Appl. Phys. 92, 3187 (2002).

${ }^{21}$ A. Cuevas, M. Kerr, and J. Schmidt, in Proceedings of the $3^{\text {rd }}$ World Conference on Photovoltaic Energy Conversion, Osaka, 2003 (2003), p. 913.

${ }^{22}$ P. P. Altermatt, H. Plagwitz, R. Bock, J. Schmidt, R. Brendel, M. J. Kerr, and A. Cuevas, in Proceedings of the $21^{\text {st }}$ European Photovoltaic Solar Energy Conference, Dresden, Germany, 2006 (WIP, Munich, 2006), p. 647.

${ }^{23}$ B. Hoex, J. Schmidt, R. Bock, P. P. Altermatt, M. C. M. van de Sanden, and W. M. M. Kessels, Appl. Phys. Lett. 91, 112107 (2007).

${ }^{24}$ S. Duttagupta, F. Lin, K. D. Shetty, A. G. Aberle, and B. Hoex, Prog. Photovolt.: Res. Appl. 21, 760 (2012)

${ }^{25}$ P. Saint-Cast, A. Richter, E. Billot, M. Hofmann, J. Benick, J. Rentsch, R. Preu, and S. W. Glunz, Thin Solid Films 522, 336 (2012).

${ }^{26}$ F.-J. Ma, S. Duttagupta, M. Peters, G. S. Samudra, A. G. Aberle, and B. Hoex, Energy Procedia 33, 104-109 (2013).

${ }^{27}$ S. Duttagupta, F.-J. Ma, S. F. Lin, T. Mueller, A. G. Aberle, and B. Hoex, IEEE J. Photovolt. 3, 1163 (2013).

${ }^{28}$ A. Richter, J. Benick, and M. Hermle, IEEE J. Photovolt. 3, 236 (2013).

${ }^{29}$ L. E. Black and K. R. McIntosh, IEEE J. Photovolt. 3, 936 (2013).

${ }^{30}$ L. E. Black, K. M. Provancha, and K. R. McIntosh, in Proceedings of the $26^{\text {th }}$ European Photovoltaic Solar Energy Conference, Hamburg, Germany, 2011 (WIP, Munich, 2011), p. 1120.

${ }^{31}$ A. Richter, S. W. Glunz, F. Werner, J. Schmidt, and A. Cuevas, Phys. Rev. B 86, 165202 (2012).
${ }^{32}$ H. Nagel, C. Berge, and A. G. Aberle, J. Appl. Phys. 86, 6218 (1999).

${ }^{33} \mathrm{~J}$. Brody, A. Rohatgi, and A. Ristow, in Proceedings of the $11^{\text {th }}$ Workshop on Crystalline Silicon Solar Cell Materials and Processes, Estes Park, Colorado, 2001 (2001), p. 163.

${ }^{34}$ D. E. Kane and R. M. Swanson, in Proceedings of the $18^{\text {th }}$ IEEE Photovoltaic Specialists Conference, Las Vegas, 1985 (IEEE, 1985), p. 578.

${ }^{35}$ K. Misiakos and D. Tsamakis, J. Appl. Phys. 74, 3293 (1993).

${ }^{36}$ W. Liang, K. J. Weber, D. Suh, S. P. Phang, J. Yu, A. K. McAuley, and B. R. Legg, IEEE J. Photovolt. 3, 678 (2013).

${ }^{37}$ B. Liao, R. Stangl, F. Ma, Z. Hameiri, T. Mueller, D. Chi, A. G. Aberle, C. S. Bhatia, and B. Hoex, J. Appl. Phys. 114, 094505 (2013).

${ }^{38}$ K. R. McIntosh and P. P. Altermatt, in Proceedings of the $35^{\text {th }}$ IEEE Photovoltaic Specialists Conference, Honolulu, 2010 (IEEE, 2010), p. 2188.

${ }^{39}$ D. Yan and A. Cuevas, J. Appl. Phys. 114, 044508 (2013).

${ }^{40}$ D. B. M. Klaassen, J. W. Slotboom, and H. C. de Graaff, Solid-State Electron. 35, 125 (1992).

${ }^{41}$ J. Dziewior and W. Schmid, Appl. Phys. Lett. 31, 346 (1977).

${ }^{42}$ M. J. Kerr and A. Cuevas, J. Appl. Phys. 91, 2473 (2002).

${ }^{43}$ Note that some authors use $S_{n 0}$ or $S_{p 0}$ to refer to what is essentially an effective surface recombination velocity including the influence of charge, identical to our $S_{\text {eff. }}$ This is because they make the implicit assumption that $Q_{f}=0$, so that $S_{\text {eff }}=S_{n 0}$ or $S_{p 0}$ from (A5). It should also be noted that $S_{n 0}$ and $S_{p 0}$ do not bear a simple relation to the parameter $S_{0}$ measured at depleted surfaces in the absence of illumination ${ }^{14}$ (for example via the pulsed MIS capacitor technique) since in the latter case the emission-related terms in (A1) become significant and only defects near the middle of the bandgap effectively contribute to recombination or generation.

${ }^{44}$ R. B. M. Girisch, R. P. Mertens, and R. F. de Keersmaecker, IEEE Trans. Electron Devices 35, 203 (1988).

${ }^{45}$ W. Shockley and W. T. Read, Phys. Rev. 87, 835 (1952).

${ }^{46}$ R. N. Hall, Phys Rev. 87, 387 (1952). 